\title{
TERNARY UNIVERSAL SUMS OF GENERALIZED PENTAGONAL NUMBERS
}

\author{
BYEONG-KWEON OH
}

\begin{abstract}
For an integer $m \geq 3$, every integer of the form $p_{m}(x)=$ $\underline{(m-2) x^{2}-(m-4) x}$ with $x \in \mathbb{Z}$ is said to be a generalized $m$-gonal number. Let $a \leq b \leq c$ and $k$ be positive integers. The quadruple $(k, a, b, c)$ is said to be universal if for every nonnegative integer $n$ there exist integers $x, y, z$ such that $n=a p_{k}(x)+b p_{k}(y)+c p_{k}(z)$. Sun proved in [16] that, when $k=$ 5 or $k \geq 7$, there are only 20 candidates for universal quadruples, which he listed explicitly and which all involve only the case of pentagonal numbers $(k=5)$. He verified that six of the candidates are in fact universal and conjectured that the remaining ones are as well. In a subsequent paper [3], Ge and Sun established universality for all but seven of the remaining candidates, leaving only $(5,1,1, t)$ for $t=6,8,9,10,(5,1,2,8)$ and $(5,1,3, s)$ for $s=7,8$ as candidates. In this article, we prove that the remaining seven quadruples given above are, in fact, universal.
\end{abstract}

\section{Introduction}

Let $m$ be any positive integer greater than two. An integer of the form $p_{m}(x)=\frac{(m-2) x^{2}-(m-4) x}{2}$ for some nonnegative integer $x$ is said to be a polygonal number of order $m$ (or $m$-gonal number). If the variable $x$ is an integer, $p_{m}(x)$ is called a generalized polygonal number of order $m$ (or generalized $m$ gonal number). From the definition every $m$-gonal number is a generalized $m$-gonal number and the converse is also true for $m=3$ or 4 . However if $m$ is greater than 4 , the set of all $m$-gonal numbers is a proper subset of the set of all generalized $m$-gonal numbers.

In 1638, Fermat asserted that every nonnegative integer can be written as a sum of $m$ polygonal numbers of order $m$. This was proved by Lagrange, Gauss and Cauchy in the cases $m=4, m=3$ and $m \geq 5$, respectively (see Chapter 1 of $[9])$.

The polygonal number theorem stated above was generalized in many directions. For example Lagrange's four square theorem was generalized to find all

Received April 20, 2010.

2010 Mathematics Subject Classification. Primary 11E12, 11E20.

Key words and phrases. generalized polygonal numbers, ternary universal sums.

This work was supported by Research Settlement Fund for the new faculty of SNU. 
quaternary quadratic forms that represent all nonnegative integers. Recently Bhargava and Hanke in [2] finally completed this problem by proving, so called, 290-theorem, which is a generalization of the Conway and Schneeberger's 15theorem (see [1]). Gauss' triangular theorem was first generalized by Liouville. To state the theorem more precisely we begin with defining the terminology universality. Let $a \leq b \leq c$ be positive integers. A ternary sum of polygonal numbers $a p_{i}(x)+b p_{j}(y)+c p_{k}(z)$ is said to be universal over $\mathbb{N}$ if the equation $n=a p_{i}(x)+b p_{j}(y)+c p_{k}(z)$ has a nonnegative integer solution $x, y, z$ for any nonnegative integer $n$. More generally if it has an integer solution $x, y, z$, it is said to be universal over $\mathbb{Z}$. The word "universal" in this paper refers to "universal over $\mathbb{Z} "$.

In 1862, Liouville determined all ternary universal sums of polygonal numbers in the case when $i=j=k=3$. When $i, j, k \in\{3,4\}$, this problem was done by Sun and his collaborators (see [4], [12] and [15]). Recently Sun gave in [16] a complete list of candidates of all possible ternary universal sums of (generalized) polygonal numbers. In particular he proved that there are at most 20 ternary universal sums over $\mathbb{Z}$ which are of the form $P_{a, b, c}^{k}(x, y, z)=a p_{k}(x)+b p_{k}(y)+c p_{k}(z)$ for $k=5$ or $k \geq 7$, and conjectured that these are all universal over $\mathbb{Z}$. They are, in fact, $k=5$ and

$$
\begin{aligned}
& (a, b, c)=(1,1, s) \quad 1 \leq s \leq 10, \quad s \neq 7, \\
& (1,2,2), \quad(1,2,3), \quad(1,2,4), \quad(1,2,6), \quad(1,2,8) \text {, } \\
& (1,3,3), \quad(1,3,4), \quad(1,3,6), \quad(1,3,7), \quad(1,3,8), \quad(1,3,9) \text {. }
\end{aligned}
$$

Guy realized in [5] that $P_{1,1,1}^{5}$ is universal over $\mathbb{Z}$ (for the complete proof, see $[16])$. In the same article [16] as above, Sun also proved the universality for the cases when $k=5$ and

$$
(a, b, c)=(1,1,2), \quad(1,1,4), \quad(1,2,2), \quad(1,2,4), \quad(1,1,5), \quad(1,3,6) .
$$

Note that the set of generalized hexagonal numbers is equal to the set of triangular numbers. Shortly after publishing this result Ge and Sun proved in [3] the universality for the cases when $k=5$ and

$$
(a, b, c)=(1,1,3), \quad(1,2,3), \quad(1,2,6), \quad(1,3,3), \quad(1,3,4), \quad(1,3,9) .
$$

Therefore it remains seven candidates of ternary sums of pentagonal numbers which are universal over $\mathbb{Z}$.

In this article we prove that these remaining seven candidates are all universal over $\mathbb{Z}$. One may easily show that $P_{1, b, c}^{5}(x, y, z)=n$ has a solution over $\mathbb{Z}$ if and only if

$$
(6 x-1)^{2}+b(6 y-1)^{2}+c(6 z-1)^{2}=24 n+b+c+1
$$

has a solution over $\mathbb{Z}$. If an integer $w$ is relatively prime to 6 , then $w$ or $-w$ is congruent to -1 modulo 6 . Therefore $P_{1, b, c}^{5}$ is universal over $\mathbb{Z}$ if and only if for every $n \in \mathbb{N}$ the ternary quadratic form

$$
x^{2}+b y^{2}+c z^{2}=24 n+b+c+1
$$


has an integer solution $x, y, z$ with $\operatorname{gcd}(x y z, 6)=1$. In many cases the problem can be reduced to the problem of representations of ternary quadratic forms without congruence condition on the solution. For example assume that $\operatorname{gcd}(b+$ $c+1,6)=1$. Then the equation (1.1) has an integer solution if and only if the equation

$$
x^{2}+b(x-6 y)^{2}+c(x-6 z)^{2}=24 n+b+c+1
$$

has an integer solution. Under this situation we use the method on the representations of ternary forms which is developed in [10]. It seems to be quite a difficult problem to determine whether or not $p_{a, b, c}^{k}$ is universal over $\mathbb{N}$ for $k \geq 5$.

The term lattice will always refer to an integral $\mathbb{Z}$-lattice on an $n$-dimensional positive definite quadratic space over $\mathbb{Q}$. The scale and the norm ideal of a lattice $L$ are denoted by $\mathfrak{s}(L)$ and $\mathfrak{n}(L)$ respectively. Let $L=\mathbb{Z} x_{1}+\mathbb{Z} x_{2}+\cdots+$ $\mathbb{Z} x_{n}$ be a $\mathbb{Z}$-lattice of rank $n$. We write

$$
L \simeq\left(B\left(x_{i}, x_{j}\right)\right)
$$

The right hand side matrix is called a matrix presentation of $L$. If $\left(B\left(x_{i}, x_{j}\right)\right)$ is diagonal, then we simply write $L \simeq\left\langle Q\left(x_{1}\right), \ldots, Q\left(x_{n}\right)\right\rangle$.

Throughout this paper, we always assume that every $\mathbb{Z}$-lattice $L$ is positive definite and is primitive in the sense that $\mathfrak{s}(L)=\mathbb{Z}$.

For any $\mathbb{Z}$-lattice $L, Q(\operatorname{gen}(L))(Q(L))$ denotes by the set of all integers that are represented by the genus of $L$ ( $L$ itself, respectively). In particular, following Kaplansky we call an integer a eligible if $a \in Q(\operatorname{gen}(L))$. For any integer $a, R(a, L)$ is denoted by the set of all vectors $x \in L$ such that $Q(x)=a$ and $r(a, L)=|R(a, L)|$.

Any unexplained notations and terminologies can be found in [8] or [13].

\section{General tools}

For a positive integer $d$ and a non-negative integer $a$, we define

$$
S_{d, a}=\left\{d n+a \mid n \in \mathbb{Z}^{+} \cup\{0\}\right\} .
$$

A $\mathbb{Z}$-lattice $L$ is called $S_{d, a}$-universal if it represents every integer in the set $S_{d, a}$. $L$ is called $S_{d, a}$-regular if it represents every integer in $S_{d, a}$ that is represented by the genus of $L$, and at least one integer in $S_{d, a}$ is represented by the genus of $L$. Hence we have

$$
L \text { is } S_{d, a} \text {-universal if and only if } S_{d, a} \subset Q(L)
$$

and

$L$ is $S_{d, a}$-regular if and only if $\varnothing \neq Q(\operatorname{gen}(L)) \cap S_{d, a} \subset Q(L)$.

For the finiteness theorem of ternary $S_{d, a}$-universal and $S_{d, a}$-regular lattices, see $[11]$. 
Let $M$ and $N$ be ternary $\mathbb{Z}$-lattices on the quadratic space $V$. For any positive integer $d$ and an integer $a$ such that $0 \leq a<d$, we define

$$
R(N, d, a)=\{x \in N / d N: Q(x) \equiv a \quad(\bmod d)\}
$$

and

$$
R(M, N, d)=\{\sigma \in O(V): \sigma(d N) \subset M\} .
$$

A coset (or, a vector in the coset) $x \in R(N, d, a)$ is said to be good (with respect to $M, N, d$ and $a$ ) if there is $\sigma \in R(M, N, d)$ such that $\sigma(\widetilde{x}) \in M$ for any $\widetilde{x} \in N$ satisfying $\widetilde{x} \equiv x(\bmod d)$. The subset of all good vectors in $R(N, d, a)$ is denoted by $R_{M}(N, d, a)$. If $R(N, d, a)=R_{M}(N, d, a)$, then we write

$$
N \prec_{d, a} M \text {. }
$$

Note that if $N \prec_{d, a} M$, then as stated in [10],

$$
S_{d, a} \cap Q(N) \subset Q(M) .
$$

Note that the converse is not true in general. One may also easily show that

(i) if $N$ is $S_{d, a}$-universal, so is $M$;

(ii) if the assumption holds for any $N \in \operatorname{gen}(M)$ and $S_{d, a} \cap Q(\operatorname{gen}(M)) \neq \varnothing$, then $M$ is $S_{d, a}$-regular;

(iii) if $M \rightarrow \operatorname{gen}(N), S_{d, a} \cap Q(\operatorname{gen}(M)) \neq \varnothing$, and $N$ is $S_{d, a}$-regular, then so is $M$.

Lemma 2.1. Let $V$ be a ternary quadratic space. For an isometry $\sigma \in O(V)$, define

$$
V_{\sigma}=\left\{x \in V: \text { there is a positive integer } k \text { such that } \sigma^{k}(x)=x\right\} .
$$

If $\sigma$ has an infinite order, then $V_{\sigma}$ is one dimensional subspace of $V$ and $\sigma(x)=$ $\operatorname{det}(\sigma) x$ for any $x \in V_{\sigma}$.

Proof. If $\sigma^{k}(x)=x$ and $\sigma^{s}(y)=y$ for some $s$ and $y \notin \mathbb{Q} x$, then the fixed space of $\sigma^{k s}$ is two dimensional and hence $\sigma^{k s}$ is a reflection, and $\sigma^{2 k s}=1$. This contradicts the fact that $\sigma$ has an infinite order. Note that every isometry $\sigma$ of a ternary quadratic space has an eigenvalue $\operatorname{det}(\sigma)$.

Corollary 2.2. Under the same notations as above, assume that there is a partition $R(N, d, a)-R_{M}(N, d, a)=P_{1} \cup \cdots \cup P_{k}$ satisfying the following properties: for each $i=1, \ldots, k$, there is $\tau_{i} \in O(V)$ such that

(i) $\tau_{i}$ has an infinite order;

(ii) $\tau_{i}(d N) \subset N$;

(iii) $\tau_{i}(x) \in N$ and $\tau_{i}(x)(\bmod d) \in P_{i} \cup R_{M}(N, d, a)$ for any $x \in P_{i}$.

Then we have

$$
\left(S_{d, a} \cap Q(N)\right)-\cup_{i=1}^{k} Q\left(z_{i}\right) \mathbb{Z}^{2} \subset Q(M),
$$

where the vector $z_{i}$ is a primitive vector in $N$ which is an eigenvector of $\tau_{i}$. 
Proof. Without loss of generality we may assume that $k=1$. Assume that $Q(x) \equiv a(\bmod d)$ for $x \in N$. If $x(\bmod d) \in R_{M}(N, d, a)$, then $Q(x)$ is represented by $M$. So we may assume that $x(\bmod d) \in R(N, d, a)-R_{M}(N, d, a)$. Then $\tau_{1}(x) \in N$ from the assumption. Hence if $\tau_{1}(x)(\bmod d) \in R_{M}(N, d, a)$, then we are done, otherwise $\tau_{1}^{2}(x) \in N$. Inductively we may assume that $\tau_{1}^{m}(x) \notin R_{M}(N, d, a)$ for any positive integer $m$. Therefore if $x$ is not an eigenvector of $\tau_{1}$, the set $\left\{\tau_{1}^{m}(x) \in N: m \geq 0\right\}$ is an infinite set by Lemma 2.1. This is a contradiction to the fact that the number of representations of any integer by a ternary form is finite.

Finding all ternary $\mathbb{Z}$-lattices satisfying $Q(\operatorname{gen}(L))=Q(L)$, which are called regular lattices, has long and rich history. In 1997 Kaplansky and his collaborators [7] provided a list of 913 candidates of primitive positive definite regular ternary lattices up to equivalence and stated that there are no others. All but 22 of the 913 candidates were verified to be regular in [7]. Recently the author proved in [10] the regularity of eight ternary lattices among the remaining 22 candidates. There are also some examples of ternary lattices satisfying $|Q(\operatorname{gen}(L))-Q(L)|=1$ (see, for example, [6]). However there are no known examples of ternary lattices such that $|Q(\operatorname{gen}(L))-Q(L)| \geq 2$ and for which $Q(L)$ is completely determined, although there are conditional results of this type in the literature. For example, under the generalized Riemann hypothesis for some Dirichlet $L$-functions, Ono and Soundararajan in [14] determined the set of all integers represented by the Ramanujan's ternary lattice $\langle 1,1,10\rangle$. The following theorem provides such an example without any assumption.

Every computation such as $R(N, d, a)$ and $R_{M}(N, d, a)$ for some $M, N, d$ and $a$ was done by the computer program MAPLE.

Theorem 2.3. For the ternary quadratic lattice $M=\langle 1\rangle \perp\left(\begin{array}{ll}9 & 3 \\ 3 & 10\end{array}\right)$,

$$
Q(M)=Q(\operatorname{gen}(M))-\left\{2 \cdot 2^{2 m}, 5 \cdot 2^{2 n}: m, n \geq 0\right\} .
$$

Proof. Note that $h(M)=3$. Let $a \in Q(\operatorname{gen}(M))$. First assume that $a$ is divisible by 3 . Since $M_{3} \simeq\left\langle 1,1,3^{4}\right\rangle, a=9 b$ for a positive integer $b$. Furthermore since $b$ is represented by $\langle 1,1,9\rangle \simeq\langle 1\rangle \perp\left(\begin{array}{ll}1 & 1 \\ 1 & 10\end{array}\right), a=9 b$ is represented by $\langle 9\rangle \perp\left(\begin{array}{ll}9 & 9 \\ 9 & 90\end{array}\right)$, which is a subform of $M$. Therefore $a$ is represented by $M$.

Assume that $a \equiv 1(\bmod 3)$. If we define $L=\langle 1,1,9\rangle$, then

$$
R(L, 3,1)=\left\{(0, \pm 1, s),( \pm 1,0, t): s, t \in \mathbb{F}_{3}\right\}
$$

and

$$
\left\{\left(\begin{array}{ccc}
0 & 1 & 0 \\
\frac{1}{3} & 0 & \frac{-1}{3} \\
0 & 0 & 1
\end{array}\right),\left(\begin{array}{ccc}
0 & 1 & 0 \\
\frac{1}{3} & 0 & \frac{1}{3} \\
0 & 0 & -1
\end{array}\right),\left(\begin{array}{ccc}
1 & 0 & 0 \\
0 & \frac{-1}{3} & \frac{1}{3} \\
0 & 0 & -1
\end{array}\right),\left(\begin{array}{ccc}
1 & 0 & 0 \\
0 & \frac{1}{3} & \frac{1}{3} \\
0 & 0 & -1
\end{array}\right)\right\} \subset R(M, L, 3) .
$$

In fact $|R(M, L, 3)|=16$, however we need only four given above. One may easily show that $R(L, 3,1)=R_{M}(L, 3,1)$ and hence $L \prec_{3,1} M$. Since $h(L)=1$, $a$ is represented by $L$ and is also represented by $M$. 
Finally assume that $a \equiv 2(\bmod 3)$. Let $N=\left(\begin{array}{ll}2 & 1 \\ 1 & 5\end{array}\right) \perp\langle 9\rangle$. Note that the class number of $N$ is one and it represents $a$. In this case we have

$$
R(N, 3,2)=\left\{(0, \pm 1, s),( \pm 1,0, t),( \pm 1, \pm 2, u): s, t, u \in \mathbb{F}_{3}\right\}
$$

and

$$
\left\{\left(\begin{array}{ccc}
-1 & 1 & 0 \\
\frac{1}{3} & \frac{2}{3} & \frac{1}{3} \\
0 & 0 & -1
\end{array}\right),\left(\begin{array}{ccc}
1 & -1 & 0 \\
\frac{-1}{3} & \frac{-2}{3} & \frac{1}{3} \\
0 & 0 & -1
\end{array}\right)\right\} \subset R(M, N, 3)
$$

Therefore we have

$$
R(N, 3,2)-R_{M}(N, 3,2)=\{( \pm 1,0,0),(0, \pm 1,0),( \pm 1, \pm 2,0)\} .
$$

Let $P_{1}=\{( \pm 1,0,0)\}, P_{2}=\{(0 \pm 1,0)\}$ and $P_{3}=\{( \pm 1, \pm 2,0)\}$, and

$$
\tau_{1}=\frac{1}{3}\left(\begin{array}{ccc}
-3 & -2 & -2 \\
0 & 1 & 4 \\
0 & -2 & 1
\end{array}\right), \tau_{2}=\frac{1}{3}\left(\begin{array}{ccc}
-2 & 0 & 5 \\
1 & 3 & -1 \\
-1 & 0 & -2
\end{array}\right), \tau_{3}=\frac{1}{3}\left(\begin{array}{ccc}
-2 & -2 & -5 \\
1 & -2 & 1 \\
-1 & -1 & 2
\end{array}\right) .
$$

Note that $\tau_{1}$ and $\tau_{2}$ satisfy all conditions in Corollary 2.2, however

$$
\tau_{3}(x) \in R_{M}(N, 3,2) \cup P_{2} \text { for any } x \in P_{3} .
$$

Hence we may still apply Corollary 2.2. Note that the primitive eigenvectors for each $\tau_{i}$ for $i=1,2$ are $(1,0,0)$ and $(0,1,0)$, respectively. Therefore if $a$ is not of the form $Q_{M}( \pm t, 0,0)=2 t^{2}$ or $Q_{M}( \pm t, \mp t, 0)=Q(0, \pm t, 0)=5 t^{2}$, then $a$ is also represented by $M$. Furthermore even if $a$ is one of those forms, $a$ is represented by $M$ except only when $r(a, N) \leq 4$. Assume that $a=2 t^{2}$ or $5 t^{2}$ for a positive integer $t$ and there is an odd prime $p \geq 5$ dividing $t$. Since

$r\left(2 p^{2}, N\right)=2 p+2-2\left(\frac{-2}{p}\right), r\left(5 p^{2}, N\right)=4 p+4-4\left(\frac{-2}{p}\right), r(125, N)=24$ by Minkowski-Siegel formula, there is a representation which is not contained in the eigenspace for both cases. Therefore $a$ is represented by $M$ if $t$ has an odd prime divisor. Finally $M_{2}$ is anisotropic and 2,5 is not represented by $M$. Hence every integer of the form $2 \cdot 2^{2 m}$ or $5 \cdot 2^{2 n}$ is not represented by $M$.

Lemma 2.4. Assume that a binary lattice $L=\langle 1, k\rangle$ represents a positive integer $p N$ for an odd prime $p$ not dividing $k$ and a positive integer $N$. If $L$ represents $p$ or $r\left(p^{2}, L\right)>2$ then there are positive integers $x, y$ not divisible by $p$ such that $x^{2}+k y^{2}=p N$.

Proof. When $L$ represents $p$, the proof was given by Jones in his unpublished Ph. D. dissertation (see also [6]). Though the proof of the remaining case is quite similar to this, we present the proof for the completeness. First define $\Phi_{p}(L)=\left\{a: x^{2}+k y^{2}=a\right.$ has an integer solution $x, y$ such that $\left.\operatorname{gcd}(x y, p)=1\right\}$. Note that if $a \in \Phi_{p}(L)$, then $a t^{2} \in \Phi_{p}(L)$ for any positive integer $t$ not divisible by $p$ and $k a \in \Phi_{p}(L)$. Assume that $S, T \in \Phi_{p}(L)$ and $S T \equiv 0(\bmod p)$. Let

$$
A^{2}+k B^{2}=S, \quad C^{2}+k D^{2}=T \quad \text { and } \quad A B C D \not \equiv 0 \quad(\bmod p) .
$$


Then $(A C \pm k B D)^{2}+k(A D \mp B C)^{2}=S T$ and at least one of $A C+k B D$ or $A C-k B D$ is not divisible by $p$. Hence $S T \in \Phi_{p}(L)$.

Now from the assumption we know that $p^{2} \in \Phi_{p}(L)$, that is, there are integers $x, y$ such that $x^{2}+k y^{2}=p^{2}$ with $\operatorname{gcd}(x y, p)=1$. Assume that $A^{2}+$ $k B^{2}=p N$ and $A \equiv B \equiv 0(\bmod p)$. If one of $A$ or $B$ is zero, then clearly $p N \in$ $\Phi_{p}(L)$. Assume that $A=p^{m} a$ and $B=p^{n} b$ and $a b \not \equiv 0(\bmod p)$. Without loss of generality we may assume that $m \geq n$. Since $\left(p^{m-n} a\right)^{2}+k b^{2}=p^{1-2 n} N$,

$$
\left(p^{m-n} a x \pm k b y\right)^{2}+k\left(p^{m-n} a y \mp b x\right)^{2}=p^{3-2 n} N .
$$

Now by choosing the sign suitably, we may assume that both terms in the left hand side are not divisible by $p$. Hence $p^{3-2 n} N \in \Phi_{p}(L)$. Therefore $p N=p^{2(n-1)} p^{3-2 n} N \in \Phi_{p}(L)$. This completes the proof.

\section{Ternary universal sums of pentagonal numbers}

In this section we prove that the remaining seven candidates of ternary sums of pentagonal numbers are universal over $\mathbb{Z}$.

(i) $p_{5}(x)+p_{5}(y)+6 p_{5}(z)$. First we show that for every eligible number $k$ of $F(x, y, z)=2 x^{2}+4 y^{2}+8 z^{2}+2 x y+2 y z, F(x, y, z)=k$ has an integral solution $(a, b, c) \in \mathbb{Z}^{3}$ with $a \not \equiv c(\bmod 2)$ unless $k \equiv 0(\bmod 4)$. Note that the class number of $F$ is 1 and hence $F(x, y, z)=k$ has an integer solution. Suppose that every solution $(a, b, c)$ satisfies $a \equiv c(\bmod 2)$. Then one may easily check that if $F(a, b, c)=k$,

$$
F\left(\frac{a-2 b-c}{2}, \frac{a+2 b+c}{2},-c\right)=k .
$$

Hence if we let $S=\frac{1}{2}\left(\begin{array}{ccc}1 & -2 & -1 \\ 1 & 2 & 1 \\ 0 & 0 & -2\end{array}\right)$, which has an infinite order, $S^{m}(a, b, c)^{t}$ is also an integer solution for any positive integer $m$. Therefore if $(a, b, c) \neq(t,-2 t, 7 t)$ for any $t \in \mathbb{Z}$, this is a contradiction by Corollary 2.2. Finally note that

$$
F(t,-2 t, 7 t)=F(2 t, 5 t, 5 t)
$$

for any $t \in \mathbb{Z}$. Hence if $k \not \equiv 0(\bmod 4)$, then such a solution exists always.

Now let $(a, b, c)$ be an integer solution with $a \not \equiv c(\bmod 2)$ of the equation $F(x, y, z)=6 n+2$ for a nonnegative integer $n$. Note that every positive integer of the form $6 n+2$ is an eligible integer of $F$. Let

$$
d=a+5 c, \quad e=-b+c, \quad f=c .
$$

Then $6 n+2=F(a, b, c)=2 d^{2}+4 e^{2}+54 f^{2}-2 d e-18 d f$. Therefore

$$
\begin{aligned}
24 n+8=4 F(a, b, c) & =8 d^{2}+16 e^{2}+216 f^{2}-8 d e-72 d f \\
& =d^{2}+(d-4 e)^{2}+6(d-6 f)^{2} .
\end{aligned}
$$

Since $d$ is odd, the integers $d, d-4 e$ and $d-6 f$ are relatively prime to 6 . Therefore $24 n+8=x^{2}+y^{2}+6 z^{2}$ has an integer solution $(x, y, z)$ with $\operatorname{gcd}(x y z, 6)=1$. 
(ii) $p_{5}(x)+p_{5}(y)+8 p_{5}(z)$. Let

$$
M=\left(\begin{array}{ccc}
4 & 2 & 2 \\
2 & 5 & 1 \\
2 & 1 & 10
\end{array}\right), \quad N=\left(\begin{array}{ccc}
5 & 1 & 2 \\
1 & 5 & -2 \\
2 & -2 & 8
\end{array}\right)
$$

Then we have $R(N, 6,5)-R_{M}(N, 6,5)=\{( \pm 2,3,0),(3, \pm 2,0)\}$. Let $P_{1}=$ $\{( \pm 2,3,0)\}, P_{2}=\{(3, \pm 2,0\}$, and

$$
\tau_{1}=\frac{1}{6}\left(\begin{array}{ccc}
-6 & -4 & -2 \\
0 & 6 & -6 \\
0 & 4 & 2
\end{array}\right), \quad \tau_{2}=\frac{1}{6}\left(\begin{array}{ccc}
-6 & 0 & -6 \\
4 & 6 & -2 \\
4 & 0 & -2
\end{array}\right) .
$$

Then one may easily show that this information satisfies all conditions in the Corollary 2.2 and the eigenvectors for each $\tau_{i}$ are $z_{1}=(1,0,0)$ and $z_{2}=(0,1,0)$. Clearly $Q\left(z_{1}\right)=Q\left(z_{2}\right)=5$ is represented by $M$. Consequently $S_{6,5} \cap Q(N) \subset$ $Q(M)$. Note that $h(N)=1, h(M)=4$ and every positive integer of the form $12 n+5$ is an eligible integer of $N$. Therefore $M$ represents every positive integer of the form $12 n+5$. Now consider the following equation

$$
\begin{aligned}
24 n+10 & =x^{2}+(x-6 y)^{2}+8(x-2 z)^{2} \\
& =10 x^{2}+36 y^{2}+32 z^{2}-12 x y-32 x z=f(x, y, z) .
\end{aligned}
$$

Since $f$ is isometric to $2 M$, the above equation has an integer solution $(x, y, z)$. Since all of $x, x-6 y, x-2 z$ are relatively prime to 6 , the equation $24 n+10=$ $x^{2}+y^{2}+8 z^{2}$ has an integer solution $(x, y, z)$ with $\operatorname{gcd}(x y z, 6)=1$.

(iii) $p_{5}(x)+p_{5}(y)+9 p_{5}(z)$. Note that every positive integer of the form $24 n+11$ is represented by $\langle 1\rangle \perp\left(\begin{array}{ll}9 & 3 \\ 3 & 10\end{array}\right)$ by Theorem 2.3 . Therefore $24 n+11=$ $x^{2}+(z-3 y)^{2}+9 z^{2}$ has always an integer solution. Note that $\operatorname{gcd}(x(z-3 y) z, 6)=$ 1.

(iv) $p_{5}(x)+p_{5}(y)+10 p_{5}(z)$. Let $M=\langle 1,4,5\rangle$ and $N=\langle 1,1,20\rangle$. Then one may easily show by computation that $N \prec_{6,0} M$. Since $\operatorname{gen}(M)=\{M, N\}$, $M$ represents every positive integer of the form $12 n+6$. Let $a, b, c$ be integers such that $12 n+6=a^{2}+4 b^{2}+5 c^{2}$. Clearly $a$ and $c$ are odd. Furthermore since $9=2^{2}+5 \cdot 1^{2}$, we may further assume that $c$ is not divisible by 3 by Lemma 2.4. Now if $x=a+2 b, y=a-2 b$ and $z=c$, then

$24 n+12=2 a^{2}+8 b^{2}+10 c^{2}=(a+2 b)^{2}+(a-2 b)^{2}+10 c^{2}=x^{2}+y^{2}+10 z^{2}$.

Since exactly one of $a$ and $b$ is divisible by $3, \operatorname{gcd}(x y z, 6)=1$.

(v) $p_{5}(x)+2 p_{5}(y)+8 p_{5}(z)$. Let

$$
M=\left(\begin{array}{ccc}
1 & 0 & 0 \\
0 & 8 & 4 \\
0 & 4 & 10
\end{array}\right), \quad N=\left(\begin{array}{ccc}
3 & 1 & 1 \\
1 & 3 & -1 \\
1 & -1 & 9
\end{array}\right), \quad L=\left(\begin{array}{ccc}
1 & 0 & 0 \\
0 & 10 & 2 \\
0 & 2 & 58
\end{array}\right)
$$

Note that $\operatorname{gen}(M)=\{M, N\}$ and $S_{24,11} \subset Q(\operatorname{gen}(M))$. Since $N \prec_{24,11} M$, $M$ represents every integer of the form $24 n+11$. Furthermore since $M \prec_{24,11}$ 
$L, L$ also represents every positive integer of that form. Therefore for any nonnegative integer $n$, there is an integer $a, b, c$ such that

$$
24 n+11=a^{2}+10 b^{2}+4 b c+58 c^{2}=a^{2}+2(b+5 c)^{2}+8(b-c)^{2} .
$$

If $x=a, y=b+5 c, z=b-c$, then one may easily show that $24 n+11=$ $x^{2}+2 y^{2}+8 z^{2}$ and $\operatorname{gcd}(x y z, 6)=1$.

(vi) $p_{5}(x)+3 p_{5}(y)+7 p_{5}(z)$. Let

$$
M=\left(\begin{array}{lll}
1 & 0 & 0 \\
0 & 3 & 0 \\
0 & 0 & 7
\end{array}\right), \quad N=\left(\begin{array}{lll}
2 & 0 & 1 \\
0 & 3 & 0 \\
1 & 0 & 4
\end{array}\right), \quad L=\left(\begin{array}{lll}
4 & 1 & 0 \\
1 & 7 & 0 \\
0 & 0 & 7
\end{array}\right) .
$$

Note that $\operatorname{gen}(M)=\{M, N\}$ and $S_{24,11} \subset Q(\operatorname{gen}(M))$. Since $N \prec_{8,3} M, M$ represents every positive integer of the form $24 n+11$. Computation shows that $R(M, 24,11)-R_{L}(M, 24,11)=\left\{(4 a, 6 b+3, \pm 4 a) \in(\mathbb{Z} / 24 \mathbb{Z})^{3}: a \not \equiv 0(\bmod 3)\right\}$.

By letting this set to $P_{1}$ and $\tau=\frac{1}{8}\left(\begin{array}{ccc}1 & 0 & 21 \\ 0 & 8 & 0 \\ -3 & 0 & 1\end{array}\right)$, we may apply Corollary 2.2 . Note that the eigenvector of $\tau$ is $(0,1,0)$, which is not in $R(N, 24,11)$. Therefore every positive integer of the form $24 n+11$ is represented by $L$. Let $a, b, c$ be integers such that

$$
24 n+11=4 a^{2}+7 b^{2}+7 c^{2}+2 a b=(a-2 b)^{2}+3(a+b)^{2}+7 c^{2} .
$$

Now let $d=a-2 b, e=a+b, f=c$. Note that def $\not \equiv 0(\bmod 3)$. By comparing both sides with modulo 8 , we have

$$
(d, e, f) \equiv(0,1,0),(1,1,1) \text { or }(0,0,1) \quad(\bmod 2) .
$$

If all of $d, e$ and $f$ are odd, then there is an integer solution $x, y, z$ such that $x^{2}+3 y^{2}+7 z^{2}=24 n+11$ with $\operatorname{gcd}(x y z, 6)=1$. Assume that $d$ and $e$ are all even. If $d=2 g, e=2 h$, then clearly $g \not \equiv h(\bmod 2)$. Furthermore

$$
d^{2}+3 e^{2}+7 f^{2}=(g \pm 3 h)^{2}+3(g \mp h)^{2}+7 f^{2} .
$$

Note that at least one of $g+h$ or $g-h$ is not divisible by 3 . Therefore there is an integer solution $x, y, z$ such that $x^{2}+3 y^{2}+7 z^{2}=24 n+11$ with $\operatorname{gcd}(x y z, 6)=1$.

Finally assume that $d$ and $f$ are all even. In this case $d \equiv f(\bmod 4)$ and $\operatorname{gcd}(e, 6)=1$. Let $d=2 i$ and $f=2 j$ and assume that $i \equiv j \equiv 1(\bmod 2)$. Then

$$
d^{2}+3 e^{2}+7 f^{2}=\left(\frac{3 i \pm 7 j}{2}\right)^{2}+3 e^{2}+7\left(\frac{i \mp 3 j}{2}\right)^{2} .
$$

Note that at least one of $\frac{i-3 j}{2}$ and $\frac{i+3 j}{2}$ is odd and both of them is not divisible by 3 . For the remaining case, that is $i \equiv j \equiv 0(\bmod 2)$, one may use the following fact to reach the same consequence: since

$$
4\left(A^{2}+7 B^{2}\right)=\left(\frac{3 A \pm 7 B}{2}\right)^{2}+7\left(\frac{A \mp 3 B}{2}\right)^{2}
$$


there are odd integers $\alpha$ and $\beta$ such that $4\left(A^{2}+7 B^{2}\right)=\alpha^{2}+7 \beta^{2}$ under the assumption that $A, B$ are all odd. If $A \not \equiv B(\bmod 2)$, then there are odd integers $\alpha^{\prime}$ and $\beta^{\prime}$ such that $16\left(A^{2}+7 B^{2}\right)=\left(\alpha^{\prime}\right)^{2}+7\left(\beta^{\prime}\right)^{2}$ (see also Lemma 3.5 of $[16])$.

(vii) $p_{5}(x)+3 p_{5}(y)+8 p_{5}(z)$. We first show that for any nonnegative integer $n$, there are integers $a, b, c$ such that

$2 n+1=a^{2}+b^{2}+2 c^{2}, b \not \equiv c(\bmod 2), a+b \not \equiv c(\bmod 3)$ and $b \not \equiv c(\bmod 3)$.

Note that $2 n+1=a^{2}+b^{2}+2 c^{2}$ has always a solution $(a, b, c) \in \mathbb{Z}^{3}$. First assume that $n$ is even. Since $\langle 1,1,8\rangle$ represents $2 n+1$ in this case, we may assume that $b$ is odd and $c$ is even. Since $b^{2}+2 c^{2} \neq 0$, we may assume that $b$ or $c$ is not divisible by 3 by Lemma 2.4. Therefore we may find integers $a, b, c$ satisfying all conditions given above by suitably choosing signs in the equation $2 n+1=( \pm a)^{2}+( \pm b)^{2}+2( \pm c)^{2}$. If $n$ is odd, we may assume that $b$ is even and $c$ is odd. Since the rest part of the proof is quite similar to the above, we are left to the readers.

Now assume that $a, b, c$ are integers satisfying the above conditions. If $d=$ $a, e=a+b+2 c, f=c-a$, then

$$
\begin{aligned}
24 n+12 & =12\left(a^{2}+b^{2}+2 c^{2}\right)=12\left(d^{2}+(-3 d+e-2 f)^{2}+2(d+f)^{2}\right) \\
& =(e-4 d)^{2}+3 e^{2}+8(e-4 d-3 f)^{2} .
\end{aligned}
$$

Note that

$$
e+f \equiv 1 \quad(\bmod 2), \quad e \not \equiv 0 \quad(\bmod 3) \text { and } d-e \not \equiv 0 \quad(\bmod 3)
$$

Therefore if $x=e-4 d, y=e, z=e-4 d-3 f$, then $24 n+12=x^{2}+3 y^{2}+8 z^{2}$ and $\operatorname{gcd}(x y z, 6)=1$.

\section{References}

[1] M. Bhargava, On the Conway-Schneeberger fifteen theorem, Quadratic forms and their applications (Dublin, 1999), 27-37, Contemp. Math., 272, Amer. Math. Soc., Providence, RI, 2000.

[2] M. Bhargava and J. Hanke, Universal quadratic forms and the 290 theorem, to appear in Invent. Math.

[3] F. Ge and Z. W. Sun, On universal sums of generalized polygonal numbers, arXiv: 0906.2450, 2009

[4] S. Guo, H. Pan, and Z. W. Sun, Mixed sums of squares and triangular numbers. II, Integers 7 (2007), A56, 5 pp.

[5] R. K. Guy, Every number is expressible as the sum of how many polygonal numbers?, Amer. Math. Monthly 101 (1994), no. 2, 169-172.

[6] W. C. Jagy, Five regular or nearly-regular ternary quadratic forms, Acta Arith. 77 (1996), no. 4, 361-367.

[7] W. C. Jagy, I. Kaplansky, and A. Schiemann, There are 913 regular ternary forms, Mathematika 44 (1997), no. 2, 332-341.

[8] Y. Kitaoka, Arithmetic of Quadratic Forms, Cambridge University Press, 1993.

[9] M. B. Nathanson, Additive Number Theory: The Classical Bases, Grad. Texts in Math. vol 164, Springer-Verlag, New York, 1991. 
[10] B.-K. Oh, Regular positive ternary quadratic forms, Acta. Arith. 147 (2011), no. 3, $233-243$.

[11] _ Representations of arithmetic progressions by positive definite quadratic forms, to appear in Int. J. Number Theory.

[12] B.-K. Oh and Z. W. Sun, Mixed sums of squares and triangular numbers. III, J. Number Theory 129 (2009), no. 4, 964-969.

[13] O. T. O'Meara, Introduction to Quadratic Forms, Springer-Verlag, New York, 1963.

[14] K. Ono and K. Soundararajan, Ramanujan's ternary quadratic form, Invent. Math. 130 (1997), no. 3, 415-454.

[15] Z. W. Sun, Mixed sums of squares and triangular numbers, Acta Arith. 127 (2007), no. $2,103-113$.

[16] _ On universal sums of polygonal numbers, arXiv:0905.0635, 2009.

Department of Mathematical Sciences and Research Institute of Mathematics Seoul National University

SeOUl 151-747, Korea

E-mail address: bkoh@snu.ac.kr 